Research Paper

\title{
Assessment of GSK1904529A as a promising anti-osteosarcoma agent
}

\author{
Hao-Dong Fei ${ }^{1, *}$, Qi Yuan ${ }^{2, *}$, Li Mao', Feng-Li Chen ${ }^{3}$, Zhao-Hui Cui ${ }^{2}$, Sha Tao ${ }^{2}$ and \\ Feng $\mathbf{J i}^{1}$ \\ ${ }^{1}$ Department of Orthopedics, Huai'an First People's Hospital, Nanjing Medical University, Huai'an, China \\ ${ }^{2}$ Department of Endocrinology, Huai'an First People's Hospital, Nanjing Medical University, Huai'an, China \\ ${ }^{3}$ Clinical Laboratory, Huai'an First People's Hospital, Nanjing Medical University, Huai'an, China \\ * Co-first authors
}

Correspondence to: Feng Ji, email: huaiaifengjidr@163.com

Li Mao, email: maoli_mail@126.com

Keywords: osteosarcoma, GSK1904529A, IGF1R, proliferation

Received: April 18, 2017 Accepted: May 04, 2017

Published: May 16, 2017

Copyright: Fei et al. This is an open-access article distributed under the terms of the Creative Commons Attribution License 3.0 (CC BY 3.0 ), which permits unrestricted use, distribution, and reproduction in any medium, provided the original author and source are credited.

\section{ABSTRACT}

The insulin growth factor-I receptor (IGF1R) signaling is a key mechanism for osteosarcoma (OS) cell proliferation. GSK1904529A is a novel small molecule IGF1R kinase inhibitor. Its activity against OS cells was tested. In both established os cell lines (Saos-2 and MG-63) and primary human OS cells, treatment with GSK1904529A (at $\mathrm{nM}$ concentrations) significantly inhibited cell proliferation. At the molecular level, GSK1904529A almost completely blocked IGF1R activation in OS cells, and inhibited downstream AKT-ERK activation. IGF1R silence by targeted shRNA also inhibited AKTERK activation and Saos-2 cell proliferation. Significantly, GSK1904529A was unable to further inhibit proliferation of IGF1R-silenced Saos-2 cells. In vivo, GSK1904529A administration orally inhibited Saos-2 tumor growth in nude mice. Together, these results suggest that targeting IGF1R by GSK1904529A inhibits OS cell growth in vitro and in vivo.

\section{INTRODUCTION}

Over the past decades, the prognosis of osteosarcoma (OS) has been improved [1-5]. The fiveyear overall survival of OS has yet reached a plateau [1-5]. The incidence of OS has been rising in the world $[6,7]$. Molecule-targeted therapy has drawn significant attentions in recent years. A better understanding of molecular pathology could likely lead to further improvements of OS treatment [8-10].

The insulin growth factor-I (IGF-I)/IGF-I receptor (IGF1R) pathway has been considered as an important contributor for OS tumorigenesis and progression [11-13]. Over-expression and/or hyper-activation of IGF1R tyrosine kinase will lead to constitutive activation of downstream signalings, including the insulin receptor substrate-1
(IRS-1) as well as AKT-ERK cascade [14, 15], which are important for a number cancerous behaviors, including cell proliferation and survival [11-13]. On the other hand, IGF1R inhibition, using genetic or pharmacological methods, could potently inhibit OS cells [11-13]. Thus, IGF1R is a valuable oncotarget protein of human OS.

Recent studies have developed GSK1904529A as a novel and potent small molecule IGF1R kinase inhibitor [16-19]. Preclinical evidences have demonstrated that GSK1904529A, by blocking IGF1R signaling, could possibly inhibit proliferation of several tumor cells [16-19]. To our best knowledge, it potential effect in human OS cells has not been studied thus far. Our results here indicate that targeting IGF1R signaling by GSK1904529A inhibits OS cell growth in vitro and in vivo. GSK1904529A could possibly have promising translational value for OS treatment. 


\section{RESULTS}

\section{GSK1904529A inhibits Saos-2 cell proliferation}

In order to study the potential effect of GSK1904529A, human OS Saos-2 cells [20, 21] were treated with GSK1904529A. Cell counting assay results in Figure 1A demonstrated that treatment GSK1904529A, at 50/250 nM, significantly inhibited Saos- 2 cell proliferation. The number of viable Saos-2 cells was significantly reduced following GSK1904529A $(50 / 250 \mathrm{nM})$ treatment, at 30-, 60-, and 90-hour durations (Figure 1A). GSK1904529A displayed a concentrationdependent manner in inhibiting Saos-2 cell proliferation (Figure 1A). Treatment with GSK1904529A in Saos-2 cells also dramatically decreased MTT OD (Figure 1B) and number of colonies (Figure 1C). GSK1904529A at $250 \mathrm{nM}$ was again more potent than $50 \mathrm{nM}$ in inhibiting Saos-2 cell proliferation (Figure 1B and 1C).

BrdU incorporation is a characteristic marker of cell proliferation. Results in Figure 1D showed that GSK1904529A treatment dose-dependently inhibited BrdU ELISA OD of Saos-2 cells, again confirming its anti-proliferative activity. Cell cycle analysis results in Figure 1E showed that GSK1904529A (250 nM) treatment induced increase of G0-G1 phase Saos-2 cells, but decrease of S phase and G2-M phase cells. Thus, GSK1904529A likely induced G1-S arrest in Saos-2 cells (Figure 1E). Collectively, these results suggest that GSK1904529A inhibits Saos-2 cell proliferation.

\section{GSK1904529A is anti-proliferative to MG-63 cells and primary human OS cells}

We also studied the effect of GSK1904529A in other OS cells. MG-63 is another wellestablished human OS cell line, which shows IGF1R-dependence [22]. MG-63 cells were treated with GSK1904529A (250 nM). As demonstrated, GSK1904529A treatment significantly decreased MTT OD (Figure 2A) and BrdU ELISA OD (Figure $2 \mathrm{~B}$ ), suggesting proliferation inhibition in MG-63 cells. Next, primary human OS cells were also treated with GSK1904529A (250 nM). Results showed that GSK1904529A also inhibited proliferation of the primary OS cells, evidence by decrease of MTT OD (Figure 2A) and BrdU ELISA OD (Figure 2B). Intriguingly, same GSK1904529A treatment was yet ineffective to OB-6 cells, which are non-cancerous human osteoblastic cells [23, 24]. MTT OD (Figure 2A) and BrdU ELISA OD (Figure 2B) were not significantly changed after GSK1904529A treatment in OB-6 cells (Figure 2A and 2B). Thus, GSK1904529A is selectively anti-proliferative to MG-63 and primary human OS cells.

\section{GSK1904529A fails to induce OS cell apoptosis}

The potential activity of GSK1904529A on OS cell apoptosis was tested. In this study, three independent apoptosis assays were applied, including caspase-3 activity assay, histone DNA apoptosis ELISA assay and TUNELnuclei staining assay. Results of these apoptosis assays showed that GSK1904529A was unable to significantly induce apoptosis in Saos-2 cells (Figure 3A-3C). Notably, Saos-2 cells were treated with GSK1904529A ( $250 \mathrm{nM})$ for 24, 48 and 72 hours, and no significant apoptosis induction was noticed in all the time-points (Figure 3A-3C). On the other hand, methotrexate treatment $(10 \mu \mathrm{M}$, 48 hours), tested here as a positive control, induced significant apoptosis in Saos-2 cells (Figure 3A-3C). TUNEL assay results in Figure 3D demonstrated that GSK1904529A ( $250 \mathrm{nM})$ also failed to activate apoptosis in MG-63 cells and primary human OS cells. No apoptosis was apparently induced in GSK1904529A (250 nM)treated OB-6 cells (Figure 3D). Collectively, these results suggest that GSK1904529A fails to induce significant apoptosis in OS cells.

\section{GSK1904529A blocks IGF1R signaling in OS cells}

GSK1904529A is a novel small-molecule inhibitor of IGF1R tyrosine kinase [16-19]. Its potential effect on IGF1R signaling was tested. Western blotting assay was performed, and quantified results in Figure 4A showed that basal IGF1R activation, tested by phosphorylated (p-) IGF1R and p-IRS-1 (insulin receptor substrate 1), was high in Saos-2 cells, which was almost completely blocked with GSK1904529A $(250 \mathrm{nM})$ treatment (Figure 4A, data were quantified). Consequently, activation of IGF1R downstream signaling, including AKT and ERK, was also largely inhibited by GSK1904529A (Figure 4B, data were quantified). Similar results were also obtained in the primary human OS cells, and GSK1904529A $(250 \mathrm{nM})$ treatment almost blocked IGF1R-IRS-1 activation (Figure 4C), causing AKT and ERK inhibition (Figure 4D). Notably, expression of total above-motioned kinases was unchanged by GSK1904529A. On the other hand, basal activation of IGF1R (p-IGF1R/p-IRS-1) was extremely low in OB-6 osteoblastic cells (Figure 4E), and treatment with GSK1904529A failed to inhibit downstream AKT and ERK activation (Figure 4F). Thus, GSK1904529A apparently blocks IGF1R signaling in OS cells.

\section{IGF1R knockdown by targeted shRNA abolishes GSK1904529A's activity in Saos-2 cells}

If IGF1R is the main target of GSK1904529A, it should be in-effective to IGF1R-depleted cells. To test this hypothesis, targeted shRNA was applied to 
knockdown IGF1R in Saos-2 cells. Western blotting assay results in Figure 5A showed that IGF1R expression was dramatically downregulated in stable Saos-2 cells with IGF1R-shRNA. Consequently, IRS-1 phosphorylation was almost completely blocked (Figure 5A). Further, downstream AKT and ERK activation was also inhibited in IGF1R-silenced Saos-2 cells (Figure 5B). Thus, IGF1R is important for downstream IRS-1, AKT and ERK activation in Saos-2 cells. Significantly, Saos-2 cell proliferation, tested by cell counting (Figure 5C) and MTT assay (Figure 5D), was also inhibited with IGF1R knockdown. Remarkably, treatment with GSK1904529A (250 nM) was unable to further inhibit proliferation of IGF1R-silenced Saos-2 cells (Figure 5C and 5D). Thus, IGF1R knockdown by targeted shRNA likely abolishes GSK1904529A's activity in Saos-2 cells.

\section{GSK1904529A administration inhibits Saos-2 tumor growth in mice}

At last, the potential anti-OS activity of GSK1904529A in vivo was tested. Saos-2 xenograft tumor model was established. A significant amount of Saos-2 OS cells were s.c. injected to the flanks of nude mice to establish xenograft tumors. Mice were then randomly assigned into three groups. GSK1904529A administration regimens were based on previous studies [17-19]. Tumor growth curve results in Figure 6A showed that GSK1904529A oral administration, at 5 and $25 \mathrm{mg} / \mathrm{kg}$ daily, significantly suppressed Saos-2 tumor growth in nude mice. GSK1904529A displayed a dose-dependent activity in vivo. GSK1904529A at $25 \mathrm{mg} / \mathrm{kg}$ was more potent than $5 \mathrm{mg} / \mathrm{kg}$ in suppressing Saos-2 tumors (Figure 6A). The results in Figure 6B demonstrated that estimated daily tumor growth, which was calculated by (estimated tumor volume at Day 50 deducting tumor volume at Day 0)/50, was also largely inhibited following GSK1904529A treatment (Figure 6B). Notably, mice body weights of different groups were not significantly different. Neither did we notice any signs of apparent toxicities.

\section{DISCUSSION}

Recent studies implied a pivotal function of IGF1R signaling in cancer progression $[13,25,26]$. Tyrosine kinase activation of IGF1R will recruit IRS-1 and other key adaptor proteins, mediating activation of downstream cascades, including PI3K-AKT-mTOR and MEK-ERK-
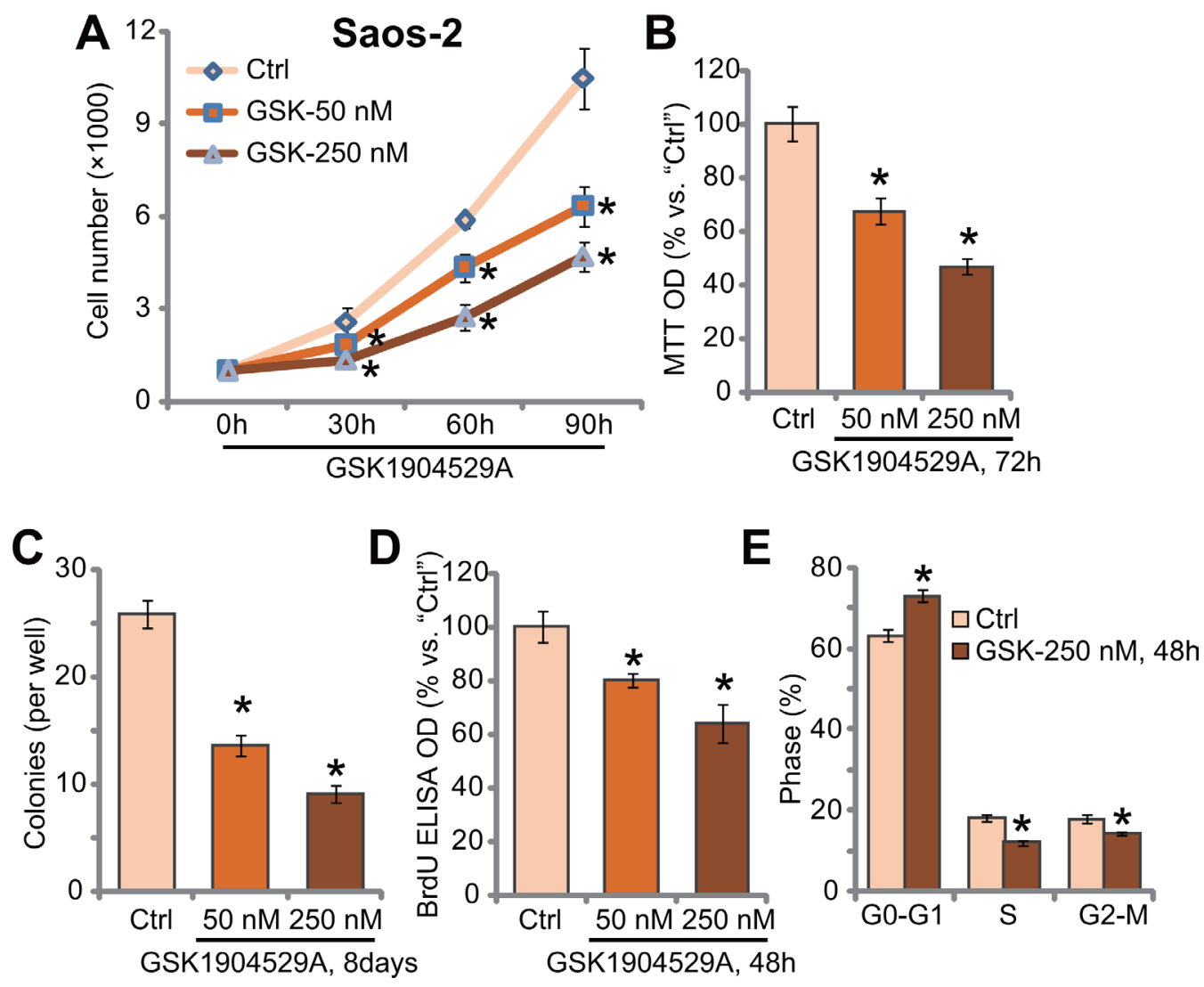

Figure 1: GSK1904529A inhibits Saos-2 cell proliferation. Saos-2 cells were either untreated ("Ctrl”, same for all Figures) or treated with GSK1904529A at designated concentration $(50 / 250 \mathrm{nM})$, cells were further maintained in the conditional medium for indicated time; Listed assays were performed to tested cell proliferation (A-E). Data were expressed as mean $\pm \mathrm{SD}(n=5)$. ${ }^{*} p<0.05 v s$. "Ctrl”. Experiments in this figure were repeated three times, and similar results were obtained. 

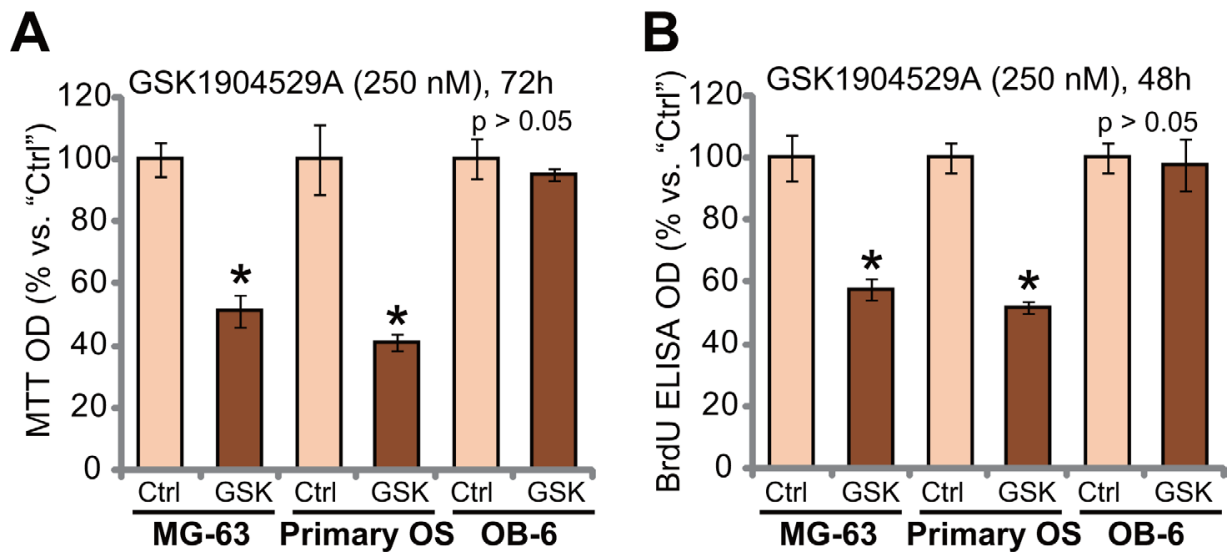

Figure 2: GSK1904529A is anti-proliferative to MG-63 cells and primary human OS cells. MG-63 cells, primary human OS cells ("Primary OS") or human OB-6 osteoblastic cells were treated with/out GSK1904529A (250 nM), cells were maintained in conditional medium for indicated time; MTT assay (A) and BrdU ELISA assay (B) were performed to test cell proliferation. Data were expressed as mean $\pm \mathrm{SD}(n=5) .{ }^{*} p<0.05 v s$. "Ctrl". Experiments in this figure were repeated three times, and similar results were obtained.
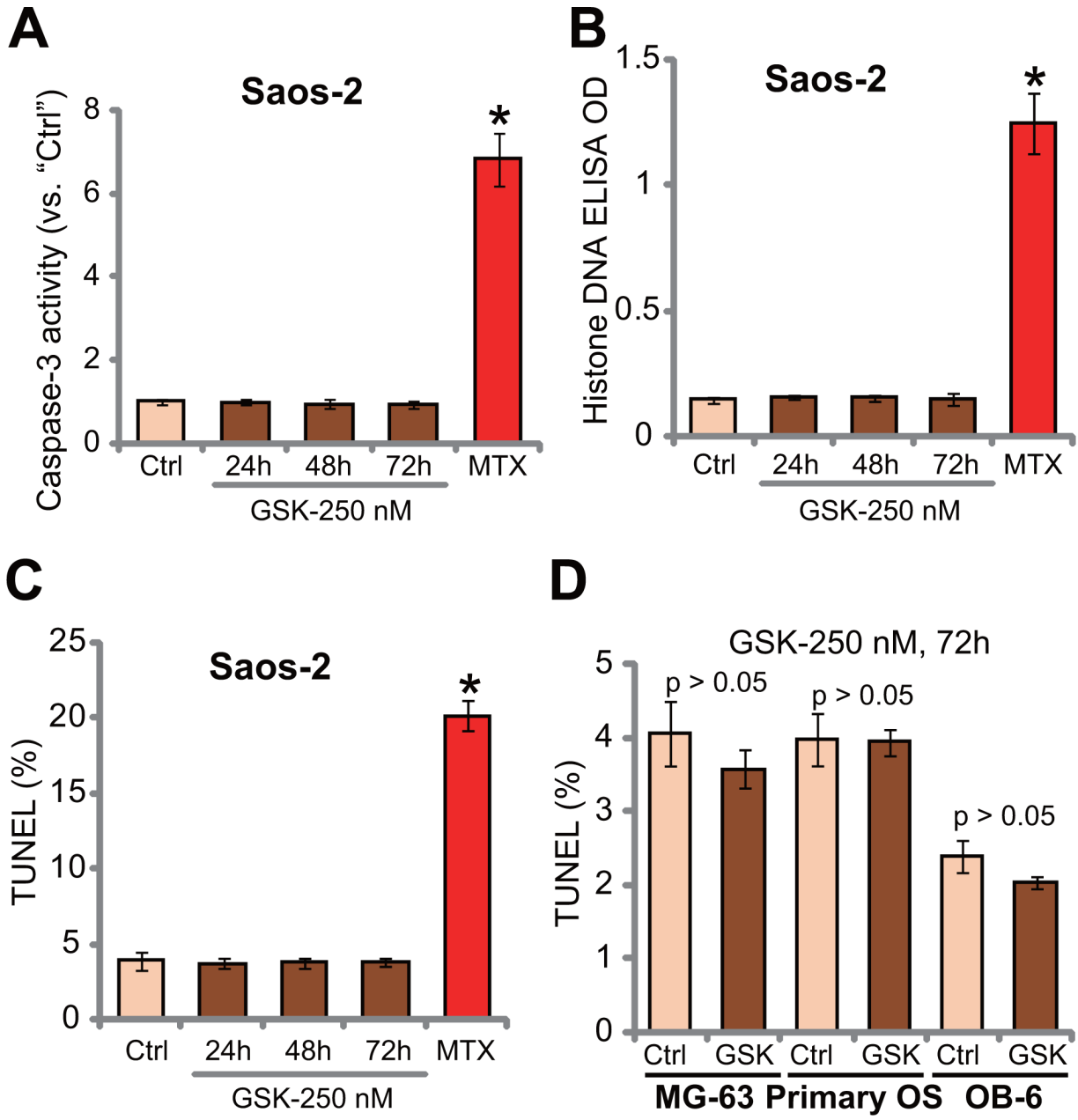

Figure 3: GSK1904529A fails to induce OS cell apoptosis. Saos-2 cells (A-C), MG-63 cells (D), primary human OS cells ("Primary OS") (D) or OB-6 osteoblastic cells (D) were treated with GSK1904529A ("GSK", $250 \mathrm{nM})$ or methotrexate (MTX, $10 \mu \mathrm{M}$ ), cells were maintained in conditional medium for indicated time; Cell apoptosis was tested by listed assays (A-D). Data were expressed as mean $\pm \mathrm{SD}(n=5) .{ }^{*} p<0.05$ vs. "Ctrl". Experiments in this figure were repeated three times, and similar results were obtained. 
MAPK signalings $[13,25,26]$. On the other hand, IGF-IR monoclonal antibodies or ATP-competitive IGF-IR inhibitors were tested, which demonstrated promising anticancer properties [13, 25, 26].

Here, we showed that GSK1904529A inhibited proliferation of established OS cell lines (Saos-2 and MG-63) and primary human OS cells. GSK1904529A almost completely blocked IGF1R activation, and inhibited downstream AKT and ERK in OS cells. In vivo, oral administration of GSK1904529A in nude mice at well-tolerated doses efficiently inhibited Saos-2 tumor growth. Thus, GSK1904529A could be further studied as a promising anti-OS agent.

It has been previously shown that serum IGF level was significantly higher in OS patients than healthy controls, which was associated with a decreased disease-free survival [22]. Meanwhile, IGF-I and IGF-II
mRNA upregulation was observed in multiple OS tumor tissues [22]. More importantly, IGR1R, insulin receptor (IR), isoform A of the insulin receptor (IR-A), as well as the IR-A/IGF1R hybrids receptors $\left(\mathrm{HR}^{\mathrm{A}}\right)$ were all upregulated in human OS tissues [22]. GSK1904529A, the ATP-competitive IGF-IR inhibitor, could possibly block both IGR1R, IRA and $\mathrm{HR}^{\mathrm{A}}[16-19,22]$. Therefore, GSK1904529A's ability to block both IGF-IR and possible other insulin receptor kinase activity potentially provides a therapeutic advantage over anti-IGF1R monoclonal antibodies.

In the current study, we indicate that IGF1R is required for GSK1904529A's activity against human OS cells. IGF1R silence by targeted shRNA mimicked GSK1904529A ability, and inhibited Saos-2 cell proliferation. More importantly, GSK1904529A was unable to further inhibit Saos-2 cells when IGF1R was
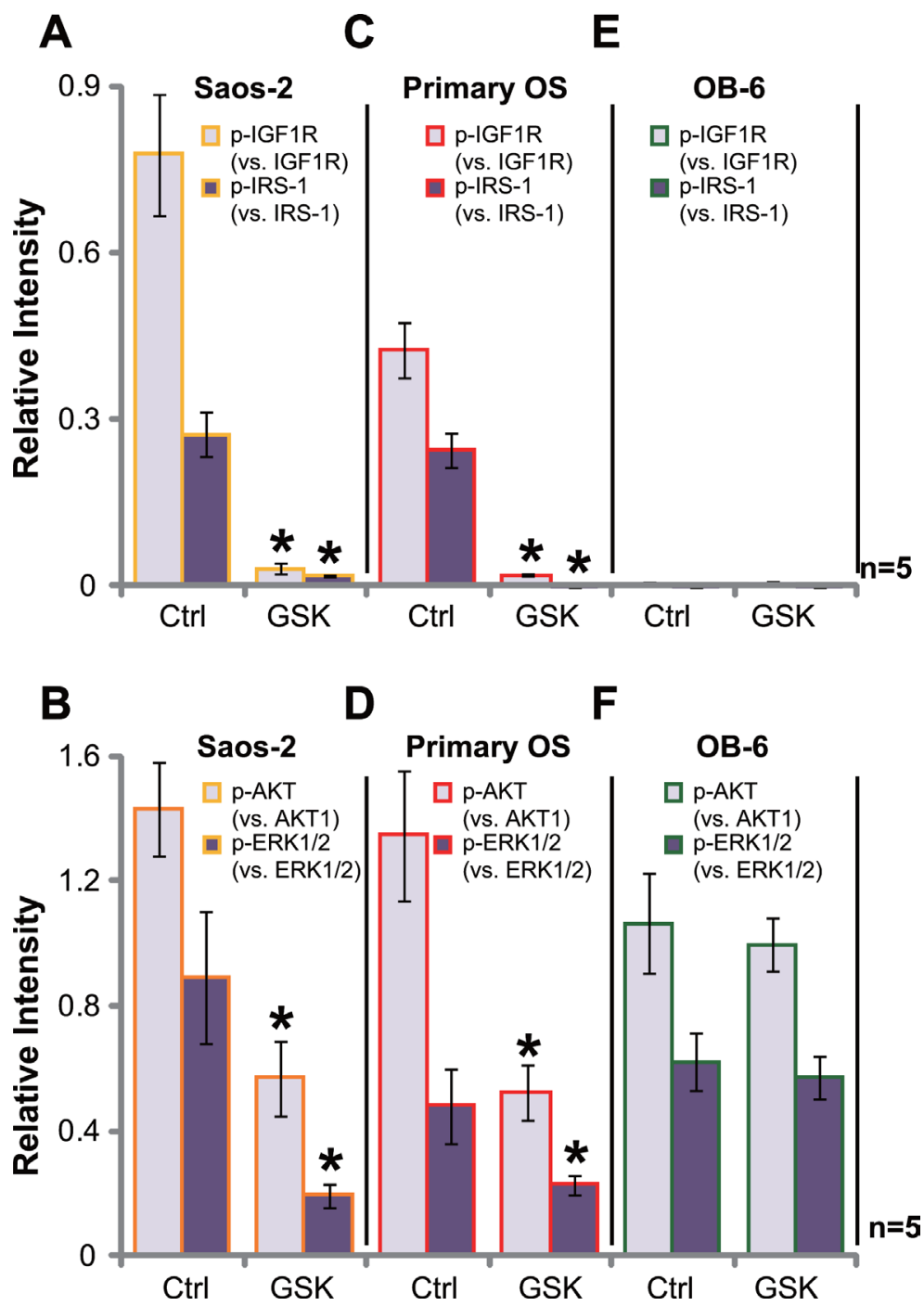

Figure 4: GSK1904529A blocks IGF1R signaling in OS cells. Saos-2 cells (A and B), primary human OS cells ("Primary OS") $(\mathbf{C}$ and $\mathbf{D})$ or OB-6 osteoblastic cells (E and F) were treated with/out GSK1904529A (250 nM) for 30 min, expressions of listed proteins were tested by Western blotting assay, data of five repeats were quantified. Data were expressed as mean $\pm \operatorname{SD}(n=5)$. * $p<0.05 v s$. "Ctrl". Experiments in this figure were repeated three times, and similar results were obtained. 
silenced. Remarkably, GSK1904529A was also ineffective to human osteoblastic OB-6 cells, where basal IGF1R activation was quite low. GSK1904529A administration in vivo was also safe to tested animals. These results suggest a selective response of GSK1904529A against activated IGF1R in OS cells.

\section{MATERIALS AND METHODS}

\section{Chemicals and reagents}

GSK1904529A was obtained from Min-de Biotech (Suzhou, China). Puromycin and methotrexate (MTX) were purchased from Sigma Aldrich (Shanghai, China). Antibodies were purchased from Cell Signaling Technology (Danvers, MA).

\section{Cell culture}

OS cell lines, including Saos-2 and MG-63, as well as human $O B-6$ [24] osteoblastic cells, were provided by the Cell Bank of the Chinese Academy of Medical Sciences (Shanghai, China). Cells were maintained in RPMI-1640 medium containing 10\% FBS (fetal bovine serum). Cell culture reagents were purchased from Gibco (Suzhou, China).

\section{Primary human OS cells}

Fresh osteoblastoma tissue (from one writteninformed patient, male, 12 years old) was minced. Tissues were then subjected to digestion with collagenase I (Sigma). Digestions 2-5 were neutralized, pooled, and filtered. Single cell suspensions of primary human OS cells were re-suspended in the described complete medium [27]. The protocol using primary human tissue was conducted with the principles expressed in the Declaration of Helsinki, and was approved by the institutional review board and Ethics Board of Nanjing Medical University.

\section{MTT assay}

MTT assay was utilized to quantify cell proliferation. Briefly, cells were initially plated in the 96well plates at $3 \times 10^{3}$ cells per well. After the designated GSK1904529A treatment, MTT dye (Sigma) was added to cells. MTT OD at $490 \mathrm{~nm}$ was recorded [28].

\section{Colony formation assay}

After indicated GSK1904529A treatment, Saos-2 cells were plated in 6-well plates at $1 \times 10^{5}$ cells per well. GSK1904529A treatment was renewed every two days for
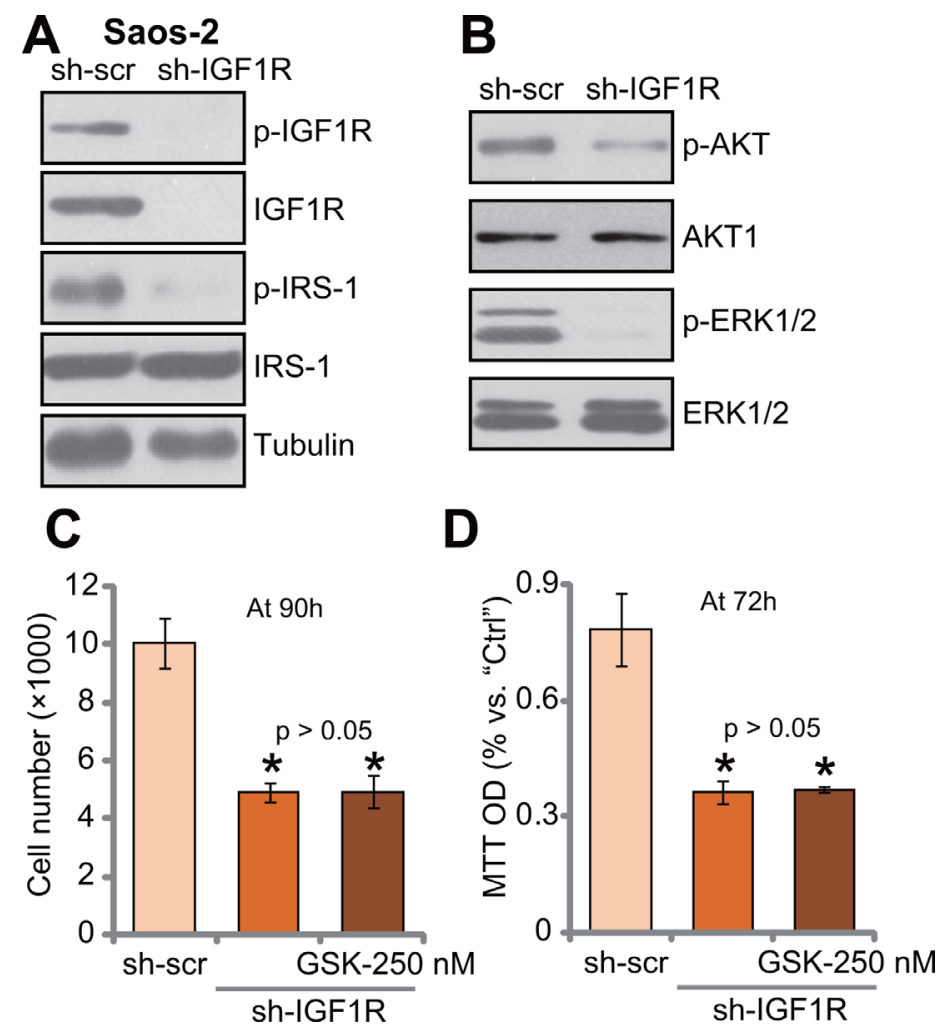

Figure 5: IGF1R knockdown by targeted shRNA abolishes GSK1904529A's activity in Saos-2 cells. Exact same amount of stable Saos-2 cells, expressing IGF1R shRNA ("sh-IGF1R") or scramble control shRNA ("sh-scr"), were subjected to Western blotting assay of listed proteins (A and B); Cells were also treated with/out GSK1904529A (250 nM) for applied time, and cell proliferation was tested by cell counting $(\mathbf{C})$ and MTT assay (D). Data were expressed as mean $\pm \operatorname{SD}(n=5)$. $* p<0.05 v s$. "sh-scr". Experiments in this figure were repeated three times, and similar results were obtained. 
a total of eight days. Afterwards, the remaining Saos-2 cell colonies were fixed, and counted.

\section{BrdU ELISA assay}

Cells with the GSK1904529A treatment were also subjected to BrdU ELISA assay kit (Cell Signaling Tech, Wuxi, China) via the attached protocol [29]. BrdU ELISA $\mathrm{OD}$ at $405 \mathrm{~nm}$ was recorded to quantify cell proliferation.

\section{Cell cycle analysis}

After the applied GSK1904529A treatment, OS cells were initially fixed by $70 \%$ ethanol. Cells were then stained with propidium iodide (PI) and subjected to FACS analysis on a Beckman Coulter flow cytometer (Suzhou, China). Cell cycle distribution was recorded.

\section{TUNEL assay of cell apoptosis}

TUNEL positive staining in cell nuclei is a characteristic marker of cell apoptosis. Cells with applied GSK1904529A treatment were subjected to TUNEL assay kit (Cell Signaling Tech) according to the attached protocol [30]. The ratio of TUNEL positive nuclei (vs. total number of nuclei) was recorded under a fluorescent microscope (Leica, Shanghai, China), summarizing at least 200 cells per condition in five independent experiments.

\section{Histone DNA apoptosis ELISA assay}

As described [31-33], Histone DNA enzyme-linked immunosorbent assay (ELISA) kit was applied to test DNA fragmentation, via the commercial available photometric sandwich immunoassay of cytoplasmic histone-associated DNA fragments (Roche, Shanghai, China).

\section{Caspase-3 activity assay}

Following the applied GSK1904529A treatment, Saos-2 cells were subjected to the Apo-ONE homogeneous caspase- 3 activity kit (Promega, Shanghai, China), which determines the caspase-3 substrate via Rhodamine 110 fluorescence. Rhodamine 110 fluorescence intensity at 500 $\mathrm{nm}$ was recorded as the caspase- 3 activity.

\section{Western blotting assay}

Protein lysates $(30 \mu \mathrm{g}$ per lane) were separated by the SDS-PAGE gels, and were transferred to PVDF (polyvinylidene difluoride) membranes. Afterwards, the blots were blocked, and incubated with designated primary and secondary antibodies. Enhanced chemiluminescence (ELC) reagents (Pierce, Shanghai, China) were applied to visualize the targeted protein band/s [32-34].

\section{IGF1R shRNA}

The lentiviral shRNA against human IGF1R was purchased from Santa Cruz Biotech (Wuxi, China), which was directly added to cultured OS cells $(10 \mu \mathrm{L} / \mathrm{mL}$, per well) for 24 hours. Puromycin $(2.5 \mu \mathrm{g} / \mathrm{mL}$, Sigma) was applied to select stable cells for 8-10 days. IGF1R silence was confirmed by Western blotting assay. Control cells were infected with lentiviral scramble control shRNA (Santa Cruz Biotech).

\section{Tumor xenograft assay}

Exponentially growing Saos-2 cells (5 million cells per mouse) were inoculated s.c. to the right flank of female nude mice (8- to 9-wk-old, 17.2-18.2 g). When the tumors reached $100 \mathrm{~mm}^{3}$ in size, mice were randomized

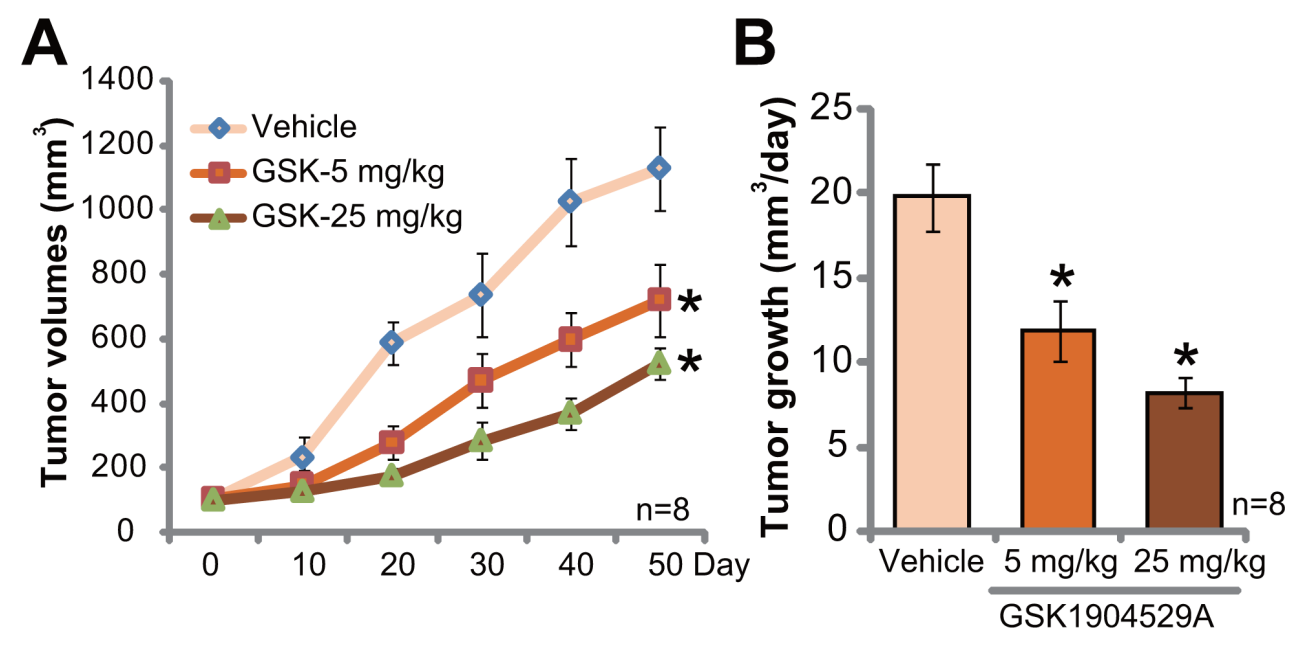

Figure 6: GSK1904529A administration inhibits Saos-2 tumor growth in mice. Saos-2 tumor-bearing nude mice were treated with GSK1904529A ( 5 or $25 \mathrm{mg} / \mathrm{kg}$ body weight, gavage, daily for 20 days) or vehicle control ("Vehicle"), tumor volumes (A) were monitored every 10 days for 50 days; Daily tumor growth was also calculated (B). Data were expressed as mean $\pm \operatorname{SD}(n=8)$. ${ }^{*} p<0.05$ vs. "Vehicle" group. 
assigned into three groups. Mice were treated orally with the vehicle or $5 / 25 \mathrm{mg} / \mathrm{kg}$ body weight of GSK1904529A, once daily for 20 consecutive days. Tumor volumes were recorded every 10 days. Tumor volume was calculated by the following formula: (length $\times$ width $\left.^{2}\right) / 2$.

\section{Statistical analysis}

The quantitative data presented in this study were mean \pm standard deviation (SD). Statistical difference was tested via one-way ANOVA with post hoc Bonferroni test.

\section{CONCLUSIONS}

In summary, the results of this preclinical study imply that targeting IGF1R signaling by GSK1904529A inhibits OS cell growth in vitro and in vivo. GSK1904529A may have significant translational value for human OS treatment.

\section{Authors' contributions}

All authors carried out the experiments, participated in the design of the study and performed the statistical analysis, participated in its design and coordination and helped to draft the manuscript.

\section{ACKNOWLEDGMENTS}

This work is supported by the National Natural Science Foundation.

\section{CONFLICTS OF INTEREST}

The listed authors have no conflicts of interests.

\section{REFERENCES}

1. Ferrari S, Serra M. An update on chemotherapy for osteosarcoma. Expert Opin Pharmacother. 2015; 16:2727-2736.

2. Ritter J, Bielack SS. Osteosarcoma. Ann Oncol. 2010; 21 Suppl 7:vii320-325.

3. Jaffe N. Osteosarcoma: review of the past, impact on the future. The American experience. Cancer Treat Res. 2009; 152:239-262.

4. Jaffe N. Adjuvant chemotherapy in osteosarcoma: an odyssey of rejection and vindication. Cancer Treat Res. 2009; 152:219-237.

5. Carrle D, Bielack SS. Current strategies of chemotherapy in osteosarcoma. Int Orthop. 2006; 30:445-451.

6. Chen W, Zheng R, Baade PD, Zhang S, Zeng H, Bray F, Jemal A, Yu XQ, He J. Cancer statistics in China, 2015. CA Cancer J Clin. 2016; 66:115-132.
7. Chen W. Cancer statistics: updated cancer burden in China. Chin J Cancer Res. 2015; 27:1.

8. Bishop MW, Janeway KA, Gorlick R. Future directions in the treatment of osteosarcoma. Curr Opin Pediatr. 2016; 28:26-33.

9. Bishop MW, Janeway KA. Emerging concepts for PI3K/ mTOR inhibition as a potential treatment for osteosarcoma. F1000Res. 2016; 5.

10. Isakoff MS, Bielack SS, Meltzer P, Gorlick R. Osteosarcoma: Current Treatment and a Collaborative Pathway to Success. J Clin Oncol. 2015; 33:3029-3035.

11. Armakolas N, Armakolas A, Antonopoulos A, Dimakakos A, Stathaki M, Koutsilieris M. The role of the IGF-1 Ec in myoskeletal system and osteosarcoma pathophysiology. Crit Rev Oncol Hematol. 2016; 108:137-145.

12. van Maldegem AM, Bovee JV, Peterse EF, Hogendoorn PC, Gelderblom H. Ewing sarcoma: The clinical relevance of the insulin-like growth factor 1 and the poly-ADP-ribosepolymerase pathway. Eur J Cancer. 2016; 53:171-180.

13. Chen HX, Sharon E. IGF-1R as an anti-cancer target--trials and tribulations. Chin J Cancer. 2013; 32:242-252.

14. MacEwen EG, Pastor J, Kutzke J, Tsan R, Kurzman ID, Thamm DH, Wilson M, Radinsky R. IGF-1 receptor contributes to the malignant phenotype in human and canine osteosarcoma. J Cell Biochem. 2004; 92:77-91.

15. Sekyi-Otu A, Bell RS, Ohashi C, Pollak M, Andrulis IL. Insulin-like growth factor 1 (IGF-1) receptors, IGF-1, and IGF-2 are expressed in primary human sarcomas. Cancer Res. 1995; 55:129-134.

16. Gupta P, Xie M, Narayanan S, Wang YJ, Wang XQ, Yuan T, Wang Z, Yang DH, Chen ZS. GSK1904529A, a Potent IGF-IR Inhibitor, Reverses MRP1-mediated Multidrug Resistance. J Cell Biochem. 2017.

17. Zhou Q, Zhang J, Cui Q, Li X, Gao G, Wang Y, Xu Y, Gao X. GSK1904529A, an insulin-like growth factor-1 receptor inhibitor, inhibits glioma tumor growth, induces apoptosis and inhibits migration. Mol Med Rep. 2015; 12:3381-3385.

18. Wang J, Qi L, Huang S, Zhou T, Guo Y, Wang G, Guo X, Zhou Z, Sha J. Quantitative phosphoproteomics analysis reveals a key role of insulin growth factor 1 receptor (IGF1R) tyrosine kinase in human sperm capacitation. Mol Cell Proteomics. 2015; 14:1104-1112.

19. Sabbatini P, Rowand JL, Groy A, Korenchuk S, Liu Q, Atkins C, Dumble M, Yang J, Anderson K, Wilson BJ, Emmitte KA, Rabindran SK, Kumar R. Antitumor activity of GSK1904529A, a small-molecule inhibitor of the insulin-like growth factor-I receptor tyrosine kinase. Clin Cancer Res. 2009; 15:3058-3067.

20. Zhu YR, Min H, Fang JF, Zhou F, Deng XW, Zhang YQ. Activity of the novel dual phosphatidylinositol 3-kinase/ mammalian target of rapamycin inhibitor NVP-BEZ235 against osteosarcoma. Cancer Biol Ther. 2015; 16:602-609.

21. Zhou R, Zhang Z, Zhao L, Jia C, Xu S, Mai Q, Lu M, Huang M, Wang L, Wang X, Jin D, Bai X. Inhibition of 
mTOR signaling by oleanolic acid contributes to its antitumor activity in osteosarcoma cells. J Orthop Res. 2011; 29:846-852.

22. Avnet S, Sciacca L, Salerno M, Gancitano G, Cassarino MF, Longhi A, Zakikhani M, Carboni JM, Gottardis M, Giunti A, Pollak M, Vigneri R, Baldini N. Insulin receptor isoform A and insulin-like growth factor II as additional treatment targets in human osteosarcoma. Cancer Res. 2009; 69:2443-2452.

23. Fan JB, Ruan JW, Liu W, Zhu LQ, Zhu XH, Yi H, Cui SY, Zhao JN, Cui ZM. miR-135b expression downregulates Ppmle to activate AMPK signaling and protect osteoblastic cells from dexamethasone. Oncotarget. 2016; 7:70613-70622. doi: 10.18632/oncotarget.12138.

24. Ding H, Wang T, Xu D, Cha B, Liu J, Li Y. Dexamethasoneinduced apoptosis of osteocytic and osteoblastic cells is mediated by TAK1 activation. Biochem Biophys Res Commun. 2015; 460:157-163.

25. Denduluri SK, Idowu O, Wang Z, Liao Z, Yan Z, Mohammed MK, Ye J, Wei Q, Wang J, Zhao L, Luu HH. Insulin-like growth factor (IGF) signaling in tumorigenesis and the development of cancer drug resistance. Genes Dis. $2015 ; 2: 13-25$.

26. Anisimov VN. Insulin/IGF-1 signaling pathway driving aging and cancer as a target for pharmacological intervention. Exp Gerontol. 2003; 38:1041-1049.

27. Zhai L, Sun N, Han Z, Jin HC, Zhang B. Liposomal shortchain C6 ceramide induces potent anti-osteosarcoma activity in vitro and in vivo. Biochem Biophys Res Commun. 2015; 468:274-280.
28. Yang L, Wu Z, Yin G, Liu H, Guan X, Zhao X, Wang J, Zhu J. Stem cell factor (SCF) protects osteoblasts from oxidative stress through activating c-Kit-Akt signaling. Biochem Biophys Res Commun. 2014; 455:256-261.

29. Zhang YM, Zhang ZQ, Liu YY, Zhou X, Shi XH, Jiang Q, Fan DL, Cao C. Requirement of Galphai1/3-Gab1 signaling complex for keratinocyte growth factor-induced PI3KAKT-mTORC1 activation. J Invest Dermatol. 2015; 135:181-191.

30. Zhou C, Chen Z, Lu X, Wu H, Yang Q, Xu D. Icaritin activates JNK-dependent $\mathrm{mPTP}$ necrosis pathway in colorectal cancer cells. Tumour Biol. 2016; 37:3135-3144.

31. Zhao S, Chen C, Wang S, Ji F, Xie Y. MHY1485 activates mTOR and protects osteoblasts from dexamethasone. Biochem Biophys Res Commun. 2016; 481:212-218.

32. Ji F, Mao L, Liu Y, Cao X, Xie Y, Wang S, Fei H. K6PC-5, a novel sphingosine kinase 1 (SphK1) activator, alleviates dexamethasone-induced damages to osteoblasts through activating SphK1-Akt signaling. Biochem Biophys Res Commun. 2015; 458:568-575.

33. Guo S, Mao L, Ji F, Wang S, Xie Y, Fei H, Wang XD. Activating AMP-activated protein kinase by an alphal selective activator compound 13 attenuates dexamethasoneinduced osteoblast cell death. Biochem Biophys Res Commun. 2016; 471:545-552.

34. Guo S, Xie Y, Fan JB, Ji F, Wang S, Fei H. alpha-Melanocyte stimulating hormone attenuates dexamethasone-induced osteoblast damages through activating melanocortin receptor 4-SphK1 signaling. Biochem Biophys Res Commun. 2016; 469:281-287. 